\title{
ANÁLISIS SOBRE LAS PROFESORAS UNIVERSITARIAS Y DESAFÍOS PARA LA PROFESIÓN ACADÉMICA EN CHILE
}

\section{Introducción}

En el proceso histórico único por el que atraviesa el sistema de educación superior, y que algunos teóricos han dado en llamar 'la era del acceso', se ve un proceso de masificación y feminización de la matrícula, su privatización y una cada vez más creciente integración internacional (IESALC, 2006). Ello trae consigo el ingreso de sectores sociales que históricamente habían estado excluidos de participar en el sistema, tales como pobres, indígenas y mujeres.

Algunas de las variables que se han utilizado para explicar la exclusión han sido la clase social o el nivel socioeconómico; sin embargo, hoy no son suficientes para explicar las transformaciones en las que se ha visto involucrado el sistema de educación superior y especialmente el universitario. Ejemplo de esto es el ingreso masivo de las mujeres ${ }^{2}$ y la creciente demanda por equidad que exige la incorporación de sectores de más bajos recursos y pueblos originarios.

¿Qué consecuencias ha tenido la incorporación de estos nuevos grupos sociales al sistema de educación superior? ¿Qué demandas y desafíos se abren a la estructura y organización interna de las universidades una vez que el perfil del estudiantado y del profesorado se ha diversificado? ¿De qué manera han de responder dichas instituciones?

2 La feminización de la matrícula que se da en gran parte de los países de América Latina, e incluso en el ámbito mundial, explicaría en gran parte la expansión de los últimos años y, por ende, el crecimiento del sistema de educación superior privado. Ver: Informe sobre Educación Superior en América Latina 2000-2005 publicado por IESALC en 2006. 


\section{Repensar las universidades y destacar el rol del profesorado universitario}

Se hace imperiosa la necesidad de que las instituciones se interroguen sobre su modo de funcionamiento a la hora de responder de manera adecuada a las exigencias y demandas sociales que se dirigen al sistema de educación superior en la actualidad. Se parte de la hipótesis de que el profesorado se convierte en un conjunto de actores privilegiados para promover la transformación de éstas hacia horizontes de mayor calidad y equidad. La situación de las profesoras universitarias reviste especial interés ya que, a pesar de que su participación en el sistema de educación superior se ha incrementado globalmente en la última década (UIS Bulletin on SET, 2006), permanecen subrepresentadas en los programas más avanzados de investigación, gestión y gobierno universitarios.

En este nivel, la desigualdad entre hombres y mujeres se expresa en el constante proceso de distinción que jerarquiza el quehacer académico y refleja tensiones en los principios y tradiciones sobre los que se funda el quehacer académico. Desde la perspectiva de género, esto lo podemos observar en el predominio masculino en la producción del conocimiento asociado al quehacer científico, en el gobierno y gestión de las instituciones de educación superior y en la producción y reproducción de la estructura patriarcal en el sistema de educación superior, que distribuye diferenciadamente a académicos y académicas según disciplinas, jerarquías y funciones.

Esbozaremos aquí sólo algunas razones estructurales de desigualdad que afectan de manera negativa a las mujeres, en su experiencia individual, académica y en las representaciones colectivas estereotipadas asociadas al género femenino y masculino que atraviesan transversalmente el trabajo académico.

\section{La perspectiva de género en el quehacer académico}

Las tensiones en el funcionamiento estructural de las instituciones de educación superior pueden ser observadas en categorías binarias, 
que se oponen y establecen jerarquías aprehendidas y reconocidas por todos los integrantes del mundo académico.

En este trabajo se ha utilizado metodología cualitativa, enfocada a la lógica de funcionamiento de las instituciones como organizaciones que reproducen los patrones de su sociedad y cultura. En el caso de la universidad, esto nos permite comprenderla como un universo de representaciones, donde las ideologías y estereotipos de género están presentes, a la vez que generan sus propias lógicas de producción y reproducción. Nos referimos a la clásica distinción simbólica entre las disciplinas, que otorga mayor valor a las ciencias exactas y aplicadas del ámbito de las ciencias naturales y matemáticas, por sobre las ciencias sociales y humanidades.

Esta distinción, largamente debatida en la epistemología y sociología del conocimiento, sitúa a las mujeres en una posición 'menos científica' ${ }^{3}$. Por otro lado, los varones están representados bajo la figura del 'científico por excelencia y tradición'4 . Las cifras corroboran esta dimensión simbólica que refleja el ámbito cultural de las instituciones, muestra el predominio de los varones en áreas de ciencias puras y tecnológicas y de las mujeres en Educación, Ciencias Sociales y Humanidades (INDICES, 2007; Araujo y Moreno, 2006; Berríos, 2005a).

Esta oposición jerárquica, que distribuye a los varones en los rangos de mayor poder y prestigio y a las mujeres -de manera mayoritaria- en las posiciones de menor poder, no implica solamente cuestiones de representación, sino que también tiene consecuencias materiales muy concretas en la desigual estructura de salarios, aun cuando puedan tener los mismas o mejores calificaciones y certificaciones educativas que sus pares varones (IESALC, 2006).

3 Asociadas en el imaginario patriarcal a lo subjetivo, las emociones, son consideradas más dispersas. Estas características son consideradas como el polo opuesto al principio de objetividad que, en el paradigma y tradición científicos, es el signo distintivo que anima la búsqueda de la ciencia.

4 En la representación colectiva aparecen en oposición a las mujeres y se considera que son capaces de enfocar su atención en un solo objeto, a la vez que se muestran como no emocionales y por tanto capaces de encarnar la objetividad. 
Una de las posibles explicaciones se funda en que las mujeres estarían menos capacitadas para tomar cargos directivos de importancia, puesto que serían un colectivo social incapaz de ejercer el mando y de tomar decisiones "duras". Las mujeres, en el imaginario colectivo patriarcal, aparecen como "mandadas" no como quienes ejercen el poder, por tanto no hay un espacio social y colectivo donde se reconozca y legitime su capacidad de ejercerlo. La universidad y el sistema de educación superior, en general, no son la excepción y tienden a reproducir la ausencia mayoritaria de mujeres en los cargos directivos y de mayor prestigio.

Otro aspecto puede ser la socialización histórica en la que se ha desenvuelto la vida de las mujeres como experiencia colectiva, donde se observa que no han sido parte de las lógicas del poder masculino que, entre otras características, circula y se reproduce a través de medios informales de toma de decisiones y establecimiento de acuerdos (citado en García de León, 1994). Las mujeres no son parte de esos circuitos informales, quedando ajenas a las instancias realmente significativas al momento de discutir y decidir las promociones del profesorado y los cargos directivos de mayor importancia en el sistema (Acker, 1995).

Los canales informales de comunicación tienen que ver con algunos ritos, como el salir a comer, conversar tomándose un café o comentar en el pasillo. Muchas mujeres se autoexcluyen de esos ritos pues no comprenden o no dimensionan su real importancia en la lógica masculina del poder. Esto se refleja en la siguiente afirmación y que viene a resumir una tendencia social común entre las mujeres:

"En este minuto tenemos una académica que está en un trabajo de asesoría directa al rector. Y tú lo ves, porque yo fui para allá a conversar, 1:30 pm, y está una cantidad de hombres vicerrectores esperando al rector. Y se van a almorzar con él. Ella se lleva su colación, porque todavía se come en la oficina" (académica universidad pública). 
Por último, la distinción faber $\mathrm{v} / \mathrm{s}$ sapiens $\mathrm{s}^{5}$, asociada a los roles de género de mujeres y hombres, tiene su correlato en el mundo académico en las distintas funciones asociadas de manera "natural" en el imaginario social, y que asume que las mujeres tienen, por ejemplo, mejores habilidades para el trabajo de archivar y ordenar, mientras que los varones más en pensar y analizar.

Este fenómeno ha sido parte de uno de los hallazgos empíricos en observaciones etnográficas sobre el trabajo científico. En un laboratorio de biología, los varones se hallaban frente al microscopio, observando y anotando sus resultados, mientras que las ayudantes estaban en las labores de orden y limpieza del laboratorio. Esta observación, que puede parecer anecdótica, no lo es si analizamos el posible desarrollo de habilidades diferenciadas entre hombres y mujeres, aprendidas a través del quehacer académico: los varones desarrollan sus capacidades de pensamiento científico, elaboran problemas y analizan resultados, expresan sus hallazgos y son capaces de generar un nuevo conocimiento; en cambio, las destrezas que desarrollan las mujeres bajo este patrón tradicional (hablamos aquí de las tendencias dominantes) están asociadas a sus roles domésticos, de apoyo y servicios, de reproductoras de un conocimiento que no generan como fruto de su propia reflexión y trabajo.

\section{Panorama de lasacadémicas en elámbito internacional}

A inicios del siglo XXI, las mujeres permanecen mayoritariamente subrepresentadas en la profesión académica en muchos lugares del mundo. El promedio mundial las sitúa en porcentajes cercanos al $30 \%$. Cifra que es significativamente inferior si las comparamos con las de participación del profesorado según sexo en los niveles básico y medio, donde son más del $80 \%$ y $50 \%$, respectivamente. Además, en este contexto, las probabilidades de tomar cargos de decisión y de mayor prestigio es menor en las mujeres que en los varones.

5 Esta idea es extraída y resumida del debate filosófico y de género sobre Homo faber v/s Homo sapiens, y al que solamente haremos alusión, pues no es nuestra intención abordar aquí esta discusión. 
El instituto de estadísticas de la UNESCO ha indicado, en promedio, un 34,8\% de mujeres dentro del cuerpo académico en la Unión Europea, en 2002. España y Suecia son los países de Europa occidental con promedios de participación femenina más altos: 41,4\% y 40,8\%, respectivamente. Alemania presenta al 2002 un 27,7\% de académicas y Dinamarca, según estadísticas de 2001, 29,9\%.

Datos del Banco Mundial (2002) señalan que en Alemania sólo un $6 \%$ de las mujeres son profesoras de tiempo completo en la más alta jerarquía de ese sistema, frente a un 29\% en Estados Unidos en las áreas de Ciencias, Ingeniería y Tecnología. En el caso del sistema francés, las mujeres son el 6,5\% del cuerpo académico titular en el área de las ingenierías y Tecnología, el 30,1\% en Humanidades, 15,3\% en Ciencias Médicas, $12,3 \%$ en Ciencias Naturales y 17\% en Ciencias Sociales.

Si bien es difícil llegar a establecer indicadores con estadísticas comparadas sobre la participación femenina mundial, dada la escasa cantidad de datos desagregados por sexo, es posible, en cambio, establecer una tendencia global que refleja claramente el gráfico 1 para el caso europeo.

Gráfico 1. Media europea para la distribución por géneros en la trayectoria de investigación.

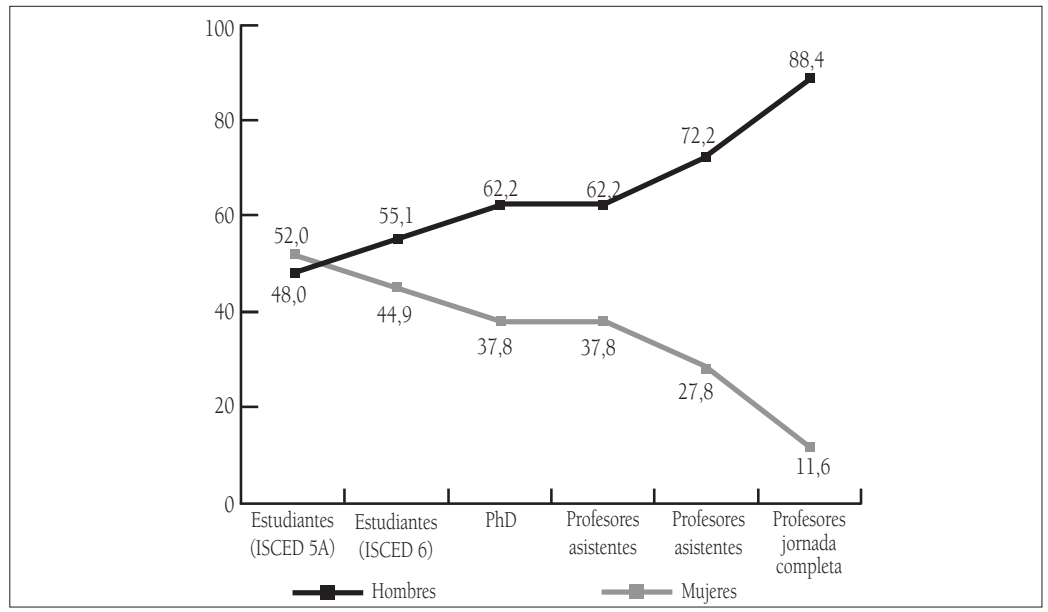

Fuente: http://ec.europa.eu/research/rtdinfo/special_rh/images/diag_211_es.jpg 
En el gráfico 1 se muestra la "pérdida" de mujeres en el sistema conforme se avanza hacia los cargos más altos de la trayectoria de investigación, según datos desagregados por sexo. Exhiben éstas una mayor participación en la matrícula de pregrado (ISCED 5A), disminuyendo progresivamente conforme se avanza en los niveles del sistema. Obtenido el grado de doctorado (Phd Graduates), los varones tienen significativamente más posibilidades (entre siete a ocho veces) de ser profesores titulares, asociados y asistentes.

Sobre los lugares en los que es posible encontrar a las académicas, un estudio señala que: "La representación espontánea de las carreras tiende a asociar las de 'letras' con un campo dominado por las mujeres y las de 'ciencias' con un ámbito plenamente masculino. Esta representación está sustentada sólo por lo que se refiere al sexo del alumnado; ahora bien, no tiene equivalencia en lo que atañe al profesorado. La relación alumnado femenino con profesorado femenino no produce la esperada supremacía de profesoras en las carreras de letras"6.

Grafico 2. \% de mujeres dentro del mundo académico en Francia, datos 2001.

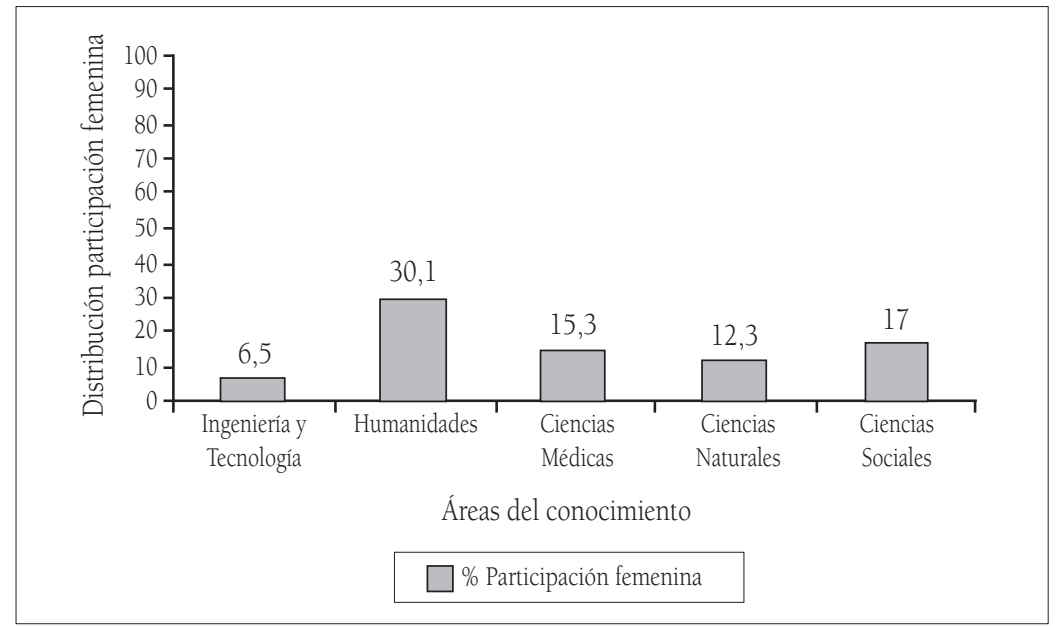

Fuente: European Commission, 2002.

6 García de León, M. Antonia (1994) Elites discriminadas (sobre el poder de las mujeres). Barcelona: Anthropos, pp. 97. 
El gráfico 2 sobre el sistema francés grafica la afirmación de la cita anterior. Nótese que, casi una década después y en los albores del siglo XXI, la situación de las mujeres no ha mejorado en términos absolutos, pese a que en el imaginario colectivo y en el discurso se las representa con las mismas posibilidades de carrera académica. Ello confirma que en el sistema de educación superior universitario opera un sistema de prestigio y poder que tiende a favorecer el desarrollo de carrera académica de los hombres por sobre el de las mujeres (Berríos, 2005b).

\section{Carrera académica en Chile: indicadores de participación femenina}

En este apartado se mostrarán algunos datos estadísticos que dan cuenta de la representación y distribución del profesorado universitario femenino y las mujeres en las planas directivas de toma de decisiones.

El siglo XX ha sido testigo privilegiado de cómo la situación descrita anteriormente se ha visto tensionada y empujada a cambiar gracias al impulso y fuerza de las demandas de los movimientos de mujeres y feministas, la transformación de la economía y el mercado laboral, los avances de la ciencia en materia de control del embarazo y la reproducción humana, y la difusión de las ideas a través de la aldea global (Castells, 1999).

Las mujeres han entrado al mundo de la educación llegando a igualar o a superar su participación en el estudiantado en relación con los varones (INDICES, 2007). Esta realidad es válida también en gran parte del continente americano y europeo, y también en muchos países de Asia.

A comienzos del siglo XXI, se puede sostener que las mujeres han ganado un espacio en el mundo público, en el mundo del trabajo y en la educación. Su ingreso masivo al mercado del trabajo ha significado adquirir progresivamente mayores niveles educativos 
y mejorar considerablemente sus niveles de empleabilidad (IESALC, 2006). Sin embargo, esos hechos no significan que el problema de la desigualdad entre hombres y mujeres se haya superado.

En nuestro caso específico, el análisis de las cifras oficiales con perspectiva de género (que no significa solamente desagregar los datos según sexo, sino que incluir nuevas variables y formas de interpretación) sostiene que la brecha más flagrante en el sistema de educación universitario opera en indicadores como el 36\% de mujeres académicas con jornada completa (INDICES, 2007), así como la baja presencia femenina en los cargos directivos superiores $(23 \%)^{7}$ que incluye rectoría, vicerrectoría y, en general, la plana directiva de investigación y posgrados.

"Si miras el organigrama ves hasta dónde llegan las mujeres. Obviamente, las mujeres tenemos techo. Creo que tenemos pocas decanas, en las secretarías del decanato sí tenemos mujeres. Y para abajo tenemos bastantes mujeres. No hay ninguna vicerrectora, jamás ha habido una rectora aquí" (académica, universidad pública).

Su techo se expresa en que no pueden acceder a ciertos cargos, mientras que sí pueden conseguirlos en los niveles más bajos de la jerarquía, sobre todo en aquellas plazas que tienen que ver con labores de tipo administrativo, como coordinador o secretario de carrera. El trabajo administrativo es un trabajo invisible, pues la relación con el alumnado no es relevante para formarse un prestigio dentro de la universidad.

El gráfico 3 muestra los porcentajes promedio de hombres y mujeres con contratos de jornada completa comparando los años 1998 y 2007. Al momento de las evaluaciones para las contrataciones, las mujeres representan sólo un tercio de éstas, en el caso del sistema

7 Según datos del Servicio Nacional de la Mujer (SERNAM), sobre la base de información del Consejo de Rectores de las Universidades Chilenas (CRUCH) a 2005. 
universitario con jornada completa. En casi diez años, si bien han aumentado en $3 \%$, las mujeres continúan siendo minorías. Si se comparan los distintos tipos de instituciones de educación superior, se puede apreciar cómo las mujeres tienen un poco más de posibilidades de tener jornada completa en los centros de formación técnica (CFT) e institutos profesionales (IP), los cuales, en comparación con el sistema universitario, poseen niveles de prestigio considerablemente menores.

Gráfico 3. \% promedio por institución docentes jornada completa Chile según sexo, años 1998 y 2007.

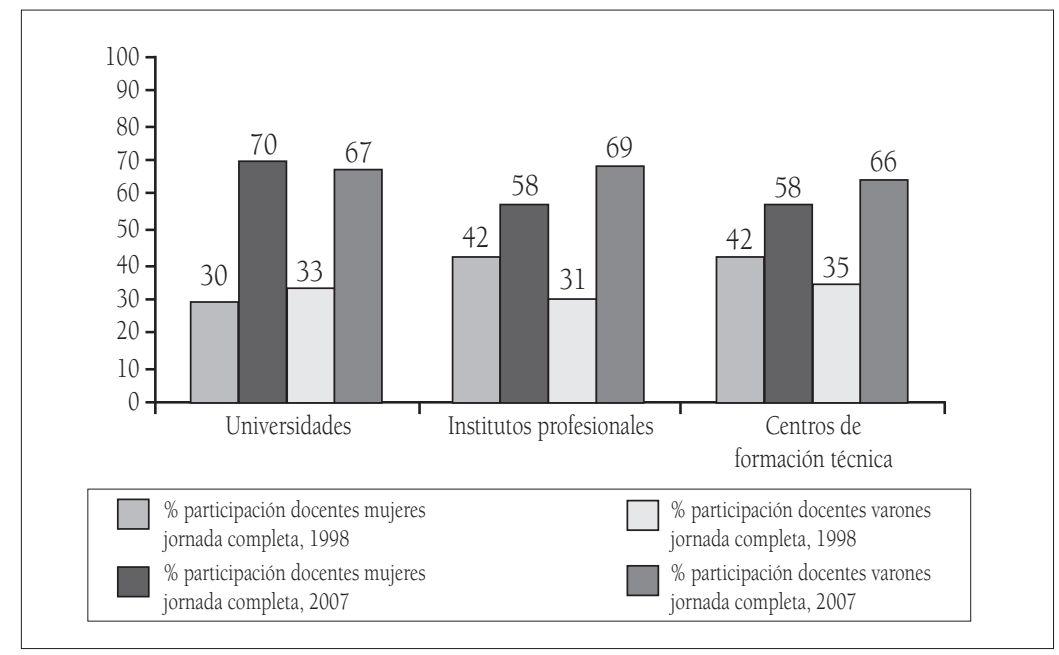

Fuente: Elaboración propia sobre la base de datos del Consejo Superior de Educación y la Corporación de Promoción Universitaria.

En el caso de las mujeres en cargos directivos en las instituciones de educación superior, en rectoría la presencia femenina en 1998 fue menor a un quinto de la proporción de hombres. En el sistema total había un 15,9\% de mujeres rectoras. Según INDICES 2007, en este año esa cifra es aún más baja, puesto que de un total de 60 universidades (públicas y privadas), 58 rectores son hombres, mientras que sólo dos son mujeres. Sólo un 3\% de mujeres llega a ejercer la máxima autoridad dentro de este sistema educativo. 
En el caso de los decanatos la tendencia también ha sido a disminuir con los años. Para el caso de las universidades del Consejo de Rectores, las mujeres decanas representan en el 2004 sólo un 6,3\%, mientras que en el año 2001 había 8,4\%. Sólo en un tercio de las universidades del CRUCH hay una decana: en la Universidad de Chile, por ejemplo.

Recordemos que en 1944 fue nombrada la primera decana de una universidad en Chile: Corina Vargas, en la Facultad de Educación de la Universidad de Concepción. Han pasado poco más de 60 años y muchas transformaciones sociales han ocurrido; sin embargo, la situación de las mujeres sigue siendo similar en el sistema de educación superior chileno. En 2004, en 8 universidades del CRUCH (de un total de 25), aún existe ese número: sólo una decana por institución.

Las cifras muestran que, a medida que se baja en la escala jerárquica, es decir, cuando se acerca más a la base del sistema de educación superior, las mujeres empiezan a estar representadas más equitativamente, siendo esto evidente en el estudiantado de pregrado que se encuentra en cifras alrededor o incluso superiores al 50\%. Es en la cúspide social y materialmente más valorada del sistema donde se presenta la diferencia más significativa entre hombres y mujeres. ¿Qué interpretaciones podemos hacer de este fenómeno?

\section{Conclusiones}

Siguiendo la interpretación de Acker (1995), podemos señalar que en el desarrollo de la carrera académica de las mujeres existen tres grandes tensiones que afectan de manera significativa su trabajo y las distancian de sus pares varones.

\section{A. Demandas conflictivas de la familia y la carrera}

Acker señala que un gran problema para ellas es cómo conciliar el trabajo con la familia. Ambas instituciones son muy demandantes y el 
dilema "surge siempre que el trabajo implica una carrera, es decir, una secuencia de promociones y ascensos por méritos; esto requiere un compromiso continuado que abarca el tiempo que, en otra situación, se dedicaría al ocio". Al igual que el trabajo doméstico, la labor académica nunca termina. Hacer una contribución a la producción de nuevos conocimientos exige un tiempo y un esfuerzo considerables. El éxito académico se mide en gran parte a través de publicaciones y para quienes tienen resuelto lo doméstico es más factible enfocar toda su energía en el trabajo tan demandante de la academia.

\section{B. Relativa indefensión de las minorías}

Este dilema aparece más evidentemente cuando la mayoría de los cargos son asumidos por varones. Ser la única mujer o una pequeña minoría dentro de un departamento universitario compuesto casi exclusivamente por varones hace ser tan invisible como visible. La invisibilidad puede transformarse, en caso extremo, en la exclusión de las redes informales de intercambio donde se toman las grandes decisiones, por ejemplo, los contratos o la promoción del profesorado. Esto es lo que García de León llama la "información informal", que operaría como una red por la que fluyen los mecanismos de poder y de la cual las mujeres rara vez forman parte.

Las minorías adquieren visibilidad en cuanto acaparan la atención por ser diferentes. ¿Qué consecuencias se siguen de esta combinación de visibilidad e invisibilidad? "Un miembro de la minoría no tiene que trabajar demasiado para que su presencia se perciba, pero sí tiene que hacerlo para que se aprecien sus logros" (Acker, 1995: 163-64).

\section{Dominio que ejercen los hombres sobre el conocimiento y la práctica}

Se refleja en los hombres que gobiernan, administran y manejan la comunidad académica, mientras que las mujeres han sido excluidas de los trabajos que producen las formas de conocimiento y las imágenes y símbolos con los que se expresa y ordena el pensamiento (Acker, 1995). 
Respecto del profesorado universitario, las mujeres siguen siendo minoría en cuanto a sus posibilidades reales de participar en instancias de mayor solvencia económica (situación contractual que presupone una jornada completa), mayor prestigio y con bases más sólidas para el desarrollo de la carrera académica que se persigue.

Las cifras comparadas de las mujeres entre 1998 y 2007 hacen plenamente vigente la necesidad de tomar en cuenta aquella recomendación de UNESCO en su visión y acción para la educación superior en el siglo XXI: "Se requieren más esfuerzos para eliminar todos los estereotipos fundados en el género en la educación superior, tener en cuenta el punto de vista del género en las distintas disciplinas, consolidar la participación cualitativa de las mujeres en todos los niveles y las disciplinas en que están insuficientemente representadas, e incrementar sobre todo su participación activa en la adopción de decisiones"8.

En relación con académicas y mujeres profesionales que se desempeñan en el sistema de educación superior, podemos señalar en definitiva que está subutilizando el capital que aportan al sistema. Se observa que las mujeres ganan menos salario que sus colegas varones; existen menos profesoras titulares (equivalencias en relación con la jornada completa); sólo alrededor de un quinto se encuentra en los cargos directivos de las respectivas instituciones, y hay más académicas en los CFT e IP que en las universidades.

Es posible observar dos grandes tendencias en las mujeres que ingresan hoy al mundo profesional académico: por un lado, tienden a casarse menos y a no tener hijos (o en escaso número); por otro, tratan de compatibilizar los roles domésticos con los académicos, siendo doble la exigencia para quienes han escogido seguir por la línea del desarrollo de carrera. Si este es el caso, eligen aquellas funciones y cargos que representen la menor demanda posible (menos

8 UNESCO (1998) La educación superior en el siglo XXI: Visión y acción. Disponible en: http:// www.unesco.org/education/educprog/wche/declaration_spa.htm Último acceso el 7 de junio de 2007. 
jornadas, más vinculadas a la docencia y administración, por sobre la investigación o el ejercicio en cargos directivos), o bien, se exigen fuertemente en términos de productividad y dedicación, restando significativamente su tiempo de ocio y descanso.

Por último, es necesario profundizar con más estudios e investigaciones sobre la vida cotidiana de las instituciones de educación superior. Se necesita más apoyo para nuevos estudios sobre las condiciones de trabajo de académicas y académicos desde la perspectiva de género. También, elaborar propuestas y estrategias de mejoramiento de la carrera académica en Chile que incorporen la perspectiva de género como parte del concepto de calidad y equidad al que debieran aspirar las instituciones educativas en el mundo de hoy.

Un punto de partida de futuras investigaciones es ¿cómo se puede llegar a construir un índice de potenciación de género en la universidad? Este aspecto "cuantitativo" debe ser complementado con la utilización de metodologías cualitativas que permitan comprender las cifras y estadísticas que existen en el sistema.

Identificar y poner en marcha áreas de cooperación y alianzas entre académicos de distintas instituciones, con el fin de promover la equidad en el desarrollo de carrera académica, debe ser uno de los más importantes desafíos para el futuro de la educación superior.

\section{Referencias bibliográficas:}

Acker, S. (1995) Género y educación. Reflexiones sociológicas sobre mujeres, enseñanza y feminismo. Madrid, Narcea ediciones.

Araujo, K. y Moreno, C. (2005) Nudos críticos para la igualdad. Género y educación superior en Chile. Santiago de Chile: Programa de Estudios de Género y Sociedad PROGÉNERO - Universidad Academia de Humanismo Cristiano.

Berríos, P. (2005a) Las profesoras universitarias de género: percepciones a partir de su experiencia académica. Tesis de Grado, Magíster en Estudios de Género y Cultura, Facultad Ciencias Sociales, Universidad de Chile. 
Berríos, P. (2005b) El sistema de prestigio en las universidades y el rol que ocupan las mujeres en el mundo académico, Calidad en la Educación, 23, pp. 349-361.

Castells, M. (1999) La era de la información. Vol. II El poder de la identidad. Madrid, Alianza Editorial.

Comisión Europea. (2003) Mujeres y ciencia. La otra mitad de la investigación. Disponible en: http://ec.europa.eu/research/rtdinfo/special_rh/print_ article_139_es.html Último acceso el 7 de junio de 2007.

García de León, Mª A. (1994) Elites discriminadas (sobre el poder de las mujeres). Barcelona: Anthropos.

IESALC (2006) Informe sobre educación superior en América Latina 2000-2005. UNESCO.

SERNAM Indicadores sobre la participación de las mujeres en educación en Chile. Disponible en: http://www.sernam.cl/basemujer/index.htm Último acceso el 7 de junio de 2007.

UNESCO (2006) Science \&Technology Bulletin, $N^{\circ} 3$, Women's participation in the research profession and different stages of higher education. Disponible en: http://www.uis.unesco.org/ev_en.php?ID=6761_201\&ID2=DO_ TOPIC Último acceso el 7 de junio de 2007.

UNESCO (1998) La educación superior en el siglo XXI: Visión y acción. Disponible en: http://www.unesco.org/education/educprog/wche/declaration_spa. htm Último acceso el 7 de junio de 2007.

WORLD BANK (2002) Constructing knowledge societies: New challenges for Tertiary Education. Education Group, Human Development Network.

Recibido: 25 de abril de 2007

Aceptado: 7 de junio de 2007 